\title{
Shale adhesion force measurements via atomic force microscopy
}

\author{
Nikolai Mitiurev $^{1, *}$, Michael Verrall ${ }^{2}$, Svetlana Shilobreeva ${ }^{3}$, Alireza Keshavarz $^{1}$, and Stefan Iglauer ${ }^{1}$ \\ ${ }^{1}$ Edith Cowan University (ECU), 270 Joondalup Drive, Joondalup, WA 6027, Australia \\ ${ }^{2}$ Commonwealth Scientific and Industrial Research Organization (CSIRO), 26 Dick Perry Ave, Kensington, WA 6151, Australia \\ ${ }^{3}$ Vernadsky Institute of Geochemistry and Analytical Chemistry of Russian Academy of Sciences, \\ Kosygin St. 19, Moscow 119991, Russia
}

Received: 18 June 2021 / Accepted: 26 October 2021

\begin{abstract}
Wettability of sedimentary rock surface is an essential parameter that defines oil recovery and production rates of a reservoir. The discovery of wettability alteration in reservoirs, as well as complications that occur in analysis of heterogeneous sample, such as shale, for instance, have prompted scientists to look for the methods of wettability assessment at nanoscale. At the same time, bulk techniques, which are commonly applied, such as USBM (United States Bureau of Mines) or Amott tests, are not sensitive enough in cases with mixed wettability of rocks as they provide average wettability values of a core plug. Atomic Force Microscopy (AFM) has been identified as one of the methods that allow for measurement of adhesion forces between cantilever and sample surface in an exact location at nanoscale. These adhesion forces can be used to estimate wettability locally. Current research, however, shows that the correlation is not trivial. Moreover, adhesion force measurement via AFM has not been used extensively in studies with geological samples yet. In this study, the adhesion force values of the cantilever tip interaction with quartz inclusion on the shale sample surface, have been measured using the AFM technique. The adhesion force measured in this particular case was equal to the capillary force of water meniscus, formed between the sample surface and the cantilever tip. Experiments were conducted with a SiconG cantilever with (tip radius of $5 \mathrm{~nm}$ ). The adhesion forces between quartz grain and cantilever tip were equal to $56.5 \pm 5 \mathrm{nN}$. Assuming the surface of interaction to be half spherical, the adhesion force per area was $0.36 \pm 0.03 \mathrm{nN} / \mathrm{nm}^{2}$. These measurements and results acquired at nano-scale will thus create a path towards much higher accuracy-wettability measurements and consequently better reservoir-scale predictions and improved underground operations.
\end{abstract}

\section{Introduction}

It is commonly acknowledged that primary recovery accounts for up to $30 \%$ of oil in place, which means that unconventional methods are required to produce the remaining hydrocarbons [1]. These methods are termed secondary and tertiary recovery, i.e., and Enhanced Oil Recovery (EOR) methods, respectively. These methods commonly apply various liquids (surfactants, low-salinity water) or gases $\left(\mathrm{CO}_{2}\right)$ in order to displace oil inside rock pore systems. Processes that occur during this displacement are defined by a number of parameters, where the most essential ones are porosity, permeability, and wettability [2].

Wettability defines whether rock pore surface tends to adhere oil, thus trapping it inside pores, or to adhere water, making the oil displacement possible and accordingly defining the pore-scale fluid flow processes. The most common techniques for estimating the wettability of rocks nowadays are USBM, Amott tests or contact angle measurements [3].

\footnotetext{
* Corresponding author: nmitiurev@gmail.com
}

However, the first two techniques require whole core plugs as samples, whereas the latter can estimate wettability only at microscale. To date, there have been numerous articles focused on this topic. For instance, Schmatz et al. [4], who have investigated pore scale fluid-fluid-solid contact between sandstone, oil and brine. However, even such advanced analytical equipment as cryo-SEM or Cryogenic Broad Ion-Beam polishing in combination with Scanning Electron Microscopy (cryo-BIB-SEM) was used to analyze the system at micrometer scale; with the bottle neck in the experiment resolution being the visual contact angle measurement. Accordingly, other scientists have conducted experiments, using contact angles of microdroplets for wettability estimation, on systems with low-salinity flooding in carbonates, i.e., limestone from Middle East to Silurian dolomite [5].

In contrast, it has been demonstrated that wettability is determined at the atomic scale through intermolecular interactions [6]. Therefore, identifying wettability at nanoscale is of great importance, where it is required to predict the behavior of pore scale interactions between surfaces of 
rock pores, oil, and fluids or gases that are used for oil displacement. Thus, in the past 20 or so years, new methods for estimating wettability more accurately at nanoscale have been developed.

AFM has emerged as a precise and accurate technique for studies at nanoscale. This technique is based on the observation of physical interactions between sample surface and tip, located at the free end of the cantilever, also known as AFM probe. These interactions can vary from raster scanning (topography imaging) to applying pressure at single point (measurement of stiffness) or measuring the adhesion forces between surface and tip. The signal, which corresponds to all above mentioned interactions is the deflection of laser, aligned to the cantilever end, which is constantly monitored by a Position Sensitive Photo-Detector (PSPD) during movement of the tip, where the signal transformed into voltage units, which can be seen in Figure 1.

Whilst initially it was mainly used to study polymer and biological molecule structures [7, 8], it has been shown to be useful to other fields of study, including measurement of adhesion forces between a cantilever and a sample surface. At first, AFM was applied by scientists to explore capillary forces in biological field of study $[9,10]$. Whilst Mori and Imae [9] investigated the adsorption process of bovine serum albumin on mica, Pericet-Camara et al. analyzed adsorption and electrostatic self-organization of poly (amidoamine) dendrimers on mica [10]. In both articles, AFM topography imaging was used due to its high resolution and ability to image nanometric structures. However, to the authors knowledge, over time these experiments did not account for reservoir rocks. For instance, [11] analyzed kerogen using AFM in a gas-shale sample, whereas [12] investigated the application of AFM for samples from Backen Formation. Yet again, these studies included only topographical images of pores, where no adhesion force measurements were included.

Only recently have articles begun to address wettability of reservoir rocks studied by AFM. To quantitatively assess wetting properties of rock surface using AFM, capillary forces of water or oil meniscus, formed between surface and cantilever tip need to be measured. To get capillary force values, one need to conduct adhesion force measurement under specific conditions [13]. In most of these papers, however, the focus has not been on studying adhesion forces at nanoscale. Only Yin and Miller conducted adhesion force measurements of kaolinite minerals and basalt planes, comparing the data to ones for talc and muscovite surfaces. The obtained results were $63 \mathrm{mN} / \mathrm{m}$ for the talc surface and $20 \mathrm{mN} / \mathrm{m}$ for kaolinite silica face [14]. Seiedi et al., on the other hand, used AFM only for topographical imaging of aged mica surfaces and calculated wettability by contact angles method [15]. At the same time, Hassenkam et al. analyzed calcite samples from water-filled zones in Maastrichtian chalk within the Danish North Sea. Although, because the authors used different measurement techniques, when the cantilever is moving perpendicular to the sample, and were dragging the cantilever across the surface instead, it is challenging to compare their results to other studies [16]. Meanwhile, quartz minerals from sandstone core in Triassic Chang-6 member of Yanchang Formation of the

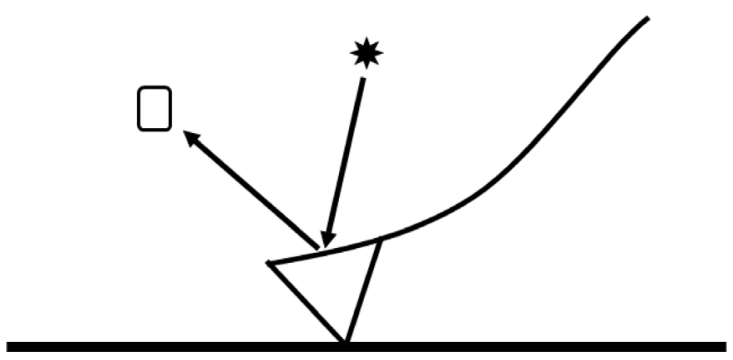

Fig. 1. Schematic representation of the AFM probe interacting with the sample surface, while laser deflection is measured by a Position Sensitive Photo-Detector (PSPD).

Ordos Basin in China have also been recently investigated. Here the authors used yet another approach to assess wettability at nanoscale. Instead of measuring the adhesion forces, they put a microdroplet on the quartz surface and conducted topography imaging of the droplet to calculate its contact angles. The measured angles varied widely from $27.8^{\circ}$ to $50.2^{\circ}$, which were higher than $25^{\circ}$, found in literature for measurements with other techniques (sessile and contact angle methods). Thus, the authors could not achieve consistent results, and, moreover, did not transfer the obtained contact angles to adhesion values [17]. Similar work has been done by [18], who calculated contact angles of water droplets at microscale on carbonate sample using Environmental Scanning Electron Microscope (ESEM). It has been shown that there are two different types of contact angles observed: $160^{\circ} \pm 3^{\circ}$ for hydrophobic and $22^{\circ} \pm 2^{\circ}$ for hydrophilic regions, which proved mixed wetting properties of carbonate surface. The most recent studies regarding this topic have been conducted by [19], who investigated the redox conditions effect on the wettability alteration of Bandera Brown sandstone samples, using AFM functionalized tips. To the authors knowledge, the closest research to the current study in terms of methodology has been done by Kumar et al. [20]. They studied wettability of aged silica and mica minerals using both AFM and contact angle measurements. Unfortunately, they do not recalculate adhesion forces per area of interaction. Moreover, they conducted experiments only for already modified mineral surfaces, thus there are no data regarding adhesion force values between original minerals and water, measured with the same cantilever.

Meanwhile, shale samples provide an even bigger challenge as a study material due to their heterogeneity and the variety of minerals they consist of [21]. With development of AFM technique as a tool to study adhesion forces, and consequently wettability at nanoscale, the analysis of each mineral on the shale sample surface became achievable. However, understanding the exact location that is analyzed at nanoscale is essential for forming the correct data interpretation. At the same time, most AFM equipment comes with a low magnification camera, which may not be suitable for the accurate positioning of the cantilever above the location of interest at nanoscale. To solve this problem, the addition of SEM and topography imaging is required. SEM allows gradual magnification, which helps 
with both locating the spots of interest within a sample, as well as with correlating the AFM camera image at mm scale with topographical image at micrometer scale. Moreover, if the surface of a shale sample is flat and polished, elemental (EDS) analysis can be conducted using SEM, thus identifying each presented mineral. Topography imaging provides information regarding the surface underneath the cantilever, which in turn allows correlation of SEM and AFM camera images with the exact location of the cantilever and, consequently, localization of the area for the adhesion force measurements.

Previously, adhesion forces between quartz and cantilever tips in the presence of humid air using AFM were assessed by Jones et al. [22]. They used $\mathrm{Si}_{3} \mathrm{~N}_{4}$ tips with 20-40 $\mathrm{nm}$ radius of curvature. Pull-off adhesion force (force, registered during cantilever retraction) was either $31 \pm 3 \mathrm{nN}$ for piranha cleaned samples, or $21 \pm 1 \mathrm{nN}$ for solvent cleaned variants. It would be incorrect, however, to compare our results to these ones, due to the significant difference in the methodology of the experiment. Indeed, they used different chemicals that could significantly modify the surface of the samples, and were pre-heating the samples to remove any water layer from them. Thus, the measuring pull-off force values seem to be artificially reduced, due to the absence of water layer between the sample and the tip.

In this paper, the adhesion force measurements of a quartz inclusion, located on the shale sample surface, were conducted using AFM. The measurements were supported by topography imaging as well as additional SEM images and EDS analysis for the shale sample surface. This paper demonstrates the importance of the combination of topography and spectroscopy experiments on AFM in order to identify the exact location for the analysis.

\section{Materials and methods}

\subsection{Sample preparation}

For the experiments a sample of carbonaceous shale from China was chosen. For the analysis, a small piece of approximately $1 \times 1 \mathrm{~mm}$ of the sample was cut from the chunk. Then the sample was mounted on a glass slide and ground down to 1500 grit size with $\mathrm{SiC}$ paper, followed by a resin bonded diamond abrasive disk with a grit size of 2000 . The sample was then polished with 3 micron, 1 micron diamonds in alcohol using resin laps, with a final $1 / 4$ micron polish using a silk lap.

\subsection{Atomic Force Microscopy}

For the experiments we used FlexAFM equipment from Nanosurf Company with Nanosurf C3000 Version 3.8.8.12 software. The cantilever used in the experiments was SiconG (Si material, Pyramidal shape of the tip with 14-16 $\mu \mathrm{m}$ height, spring constant -0.13 to $0.6 \mathrm{~N} / \mathrm{m}$, $450 \times 49 \times 2.5 \mu \mathrm{m}$ length $\times$ width $\times$ height, $10 / 50 \mathrm{~nm}$ $\mathrm{Ti} / \mathrm{Au}$ reflex side coating). Ultrahigh resolution SEM images of the cantilever tip are shown in Figure 2. As the experiment was conducted at ambient conditions, both cantilever

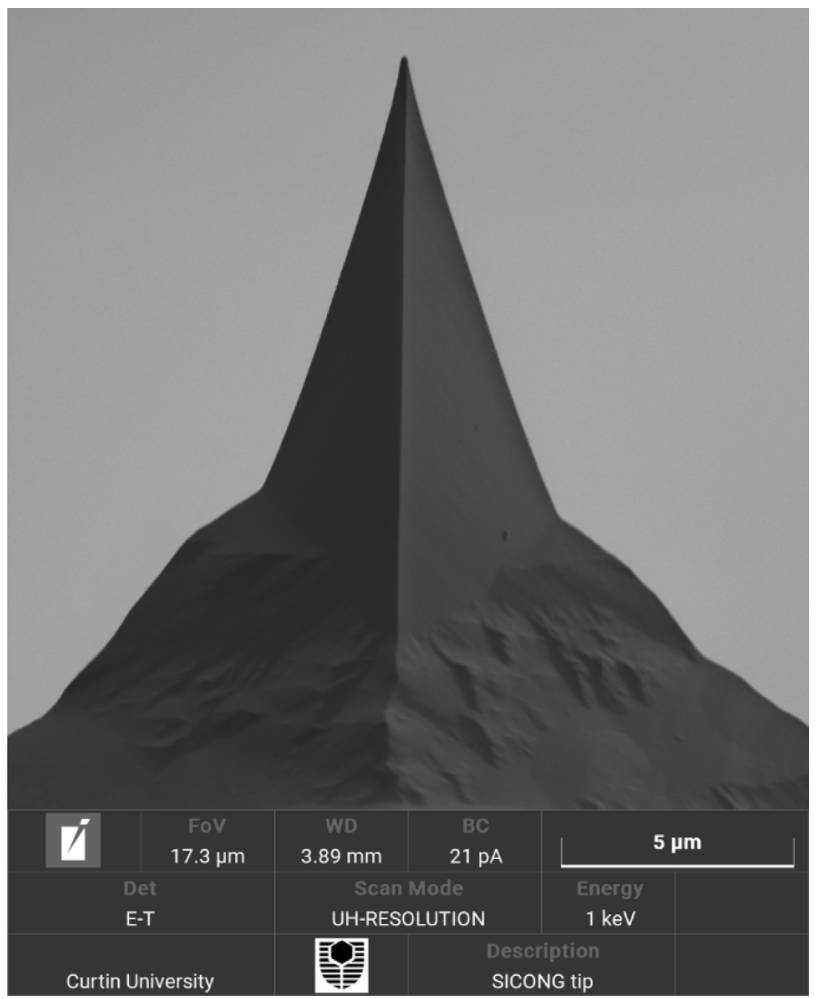

Fig. 2. FESEM image of the cantilever tip.

and sample surface were covered in water (which was adsorbed from the air).

At the start of the experiment, the sample was placed on the AFM stage, after which the cantilever was placed into the cantilever holder and the red laser was aligned to the tip of the cantilever. Topography images were taken with: an image size of $5 \times 5 \mu \mathrm{m}$, with force setpoint of $40 \mathrm{nN}$, with P-gain of 2500, and with I-gain of 700 . Prior to conducting the experiment, the $X Y$ slope was calibrated. The resolution of the final image was set to $512 \times 512$ points with $780 \mathrm{~ms}$ for each line. Area roughness was $10.5 \mathrm{~nm}$, while difference between minimum and maximum height was equal to $71 \mathrm{~nm}$.

After the location for analysis was chosen based on the surface topography, the spectroscopy mode was selected and nine spots for the adhesion force measurements were chosen on the topography image, which can be seen in Figure 3 .

\subsection{Scanning Electron Microscopy}

SEM images were obtained using a Tescan Mira3 Field Emission Scanning Electron Microscope (FESEM) fitted with an Oxford X-Max 50 energy EDS. The FESEM was operated in low vacuum mode with a chamber pressure of $40 \mathrm{~Pa}$ and at an accelerating voltage of $25 \mathrm{kV}$ with a beam current of approximately $6 \mathrm{nA}$. The EDS map was collected with $21 \mu$ s dwell time and half micron pixel spacing over a $1 \times 1 \mathrm{~mm}$ area. Ultrahigh resolution SEM images of the cantilever tip were obtained using a Tescan Clara FESEM at $17.3 \mu \mathrm{m}$ Field Of View (FOV), with $3.89 \mathrm{~mm}$ working 


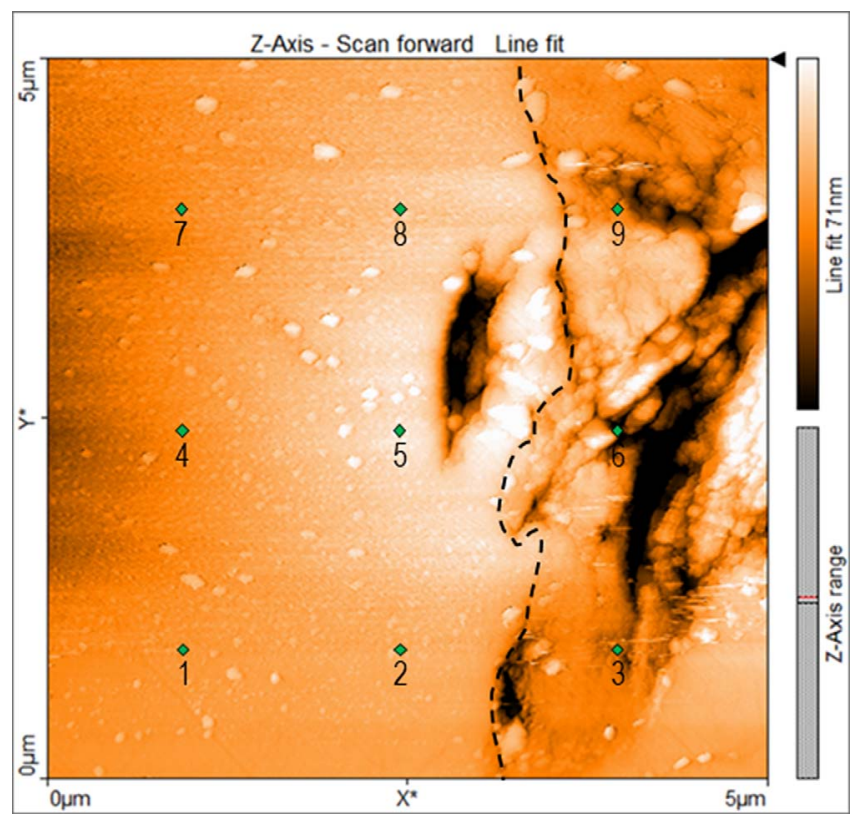

Fig. 3. Topography $(5 \times 5 \mu \mathrm{m}$ size $)$ image of a quartz grain. Nine points indicate adhesion force measurement locations. The dashed line represents contact between quartz inclusion (to the right from the dashed line) and clay (to the left).

distance (WD) and a $12 \mathrm{pA}$ beam current with $1 \mathrm{keV}$ energy.

\section{Results and discussion}

As has been mentioned in the Section 2, SEM images of the sample surface were obtained using FESEM. As can be seen in Figure 4 the surface appears heterogenous and consists of different minerals. Therefore, adhesion force values as measured at nanoscale, vary depending on which mineral is examined.

These minerals, following XRD analysis conducted on the shale sample powder and as shown in [23], were: 33.9 wt.\% quartz, 29.7 wt.\% muscovite, 21.8 wt.\% illite, 6.2 wt.\% chlorite, 5.5 wt.\% dickite, 1.5 wt.\% rutile and 1.4 wt.\% albite. There was, however, further need to identify the mineral grains on the surface, as required for accurate wettability measurements. For this purpose, EDS analysis was performed, where an elemental map of mineral grains is shown in Figure 5.

Aside from the EDS map, several spectra of both different spots and of the entire map were also measured. The one for the entire map can be seen in Figure 6. Taking into account both EDS map and spectra we confirmed the composition of our polished sample mounted on glass and the sample powder to be identical.

Therefore, based on the obtained elemental composition of the surface we decided to measure adhesion forces on a quartz grain, as it has relatively homogeneous structure. After alignment of the cantilever, topography images of $5 \times 5 \mu \mathrm{m}$ scale were collected. As can be seen from Figure 3,

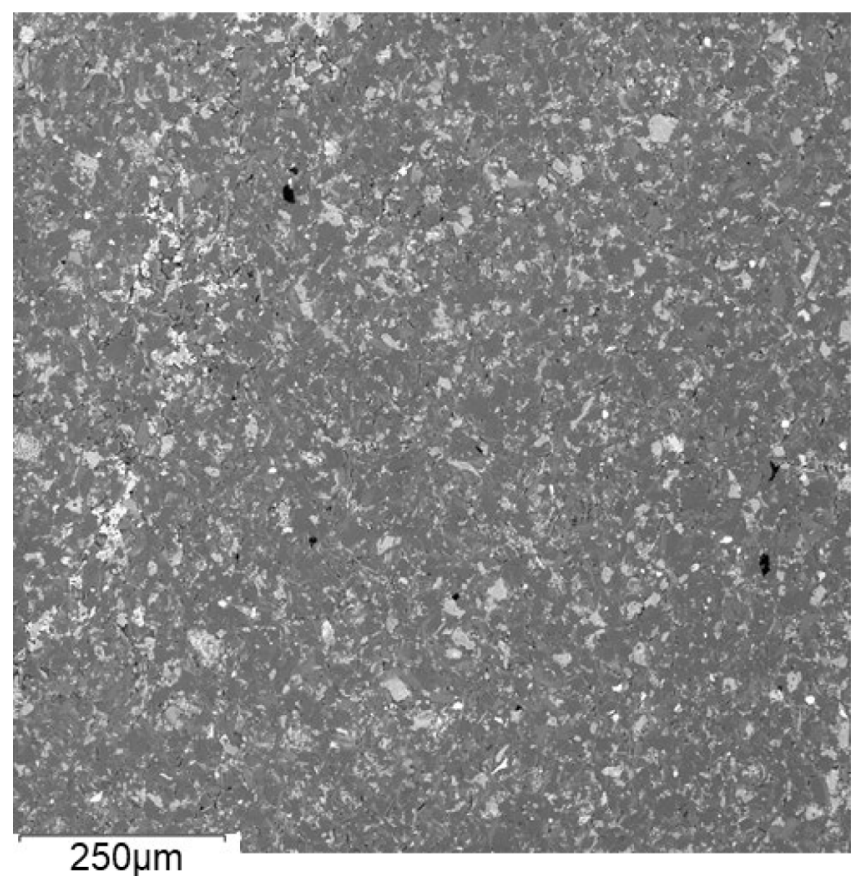

Fig. 4. SEM image of the shale sample.

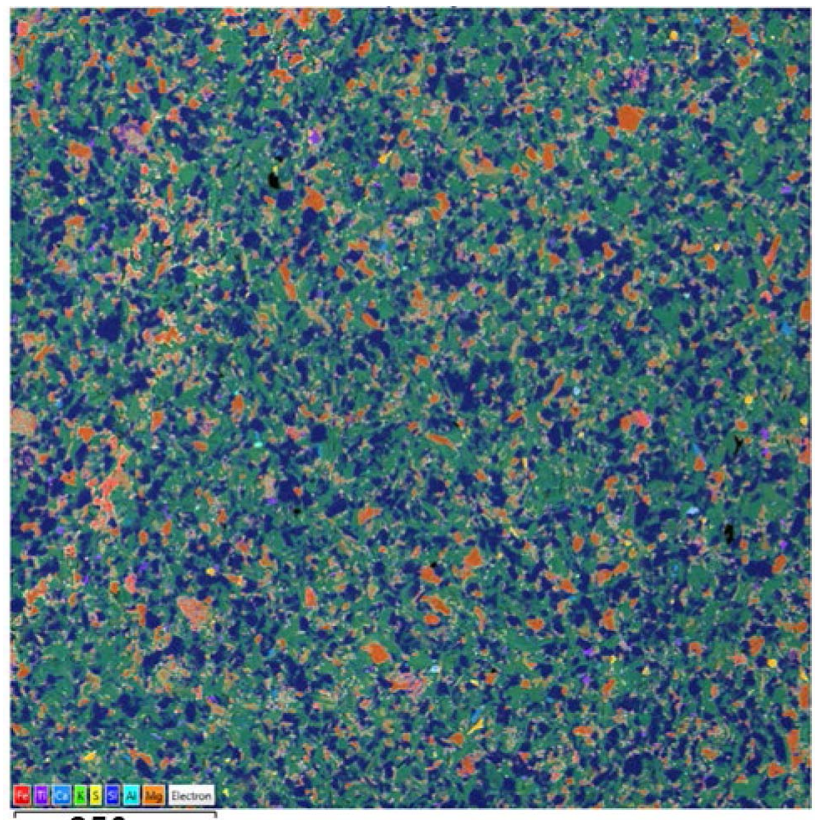

$250 \mu \mathrm{m}$

Fig. 5. EDS map of the shale surface. Fe - red, Ti - purple, Ca blue, $\mathrm{K}$ - salad green, $\mathrm{S}$ - yellow, $\mathrm{Si}$ - dark blue, $\mathrm{Al}$ aquamarine, $\mathrm{Mg}$ - orange. The dark green phase here is a combination of $\mathrm{Al}, \mathrm{K}$ and $\mathrm{Si}$ signals, which indicates muscovite mineral. The analysis shows high heterogeneity of the shale sample.

part of the polished quartz grain is on the left side of the image. However, without alignment of topography and spectroscopy scales, it is not possible to ensure that adhesion 


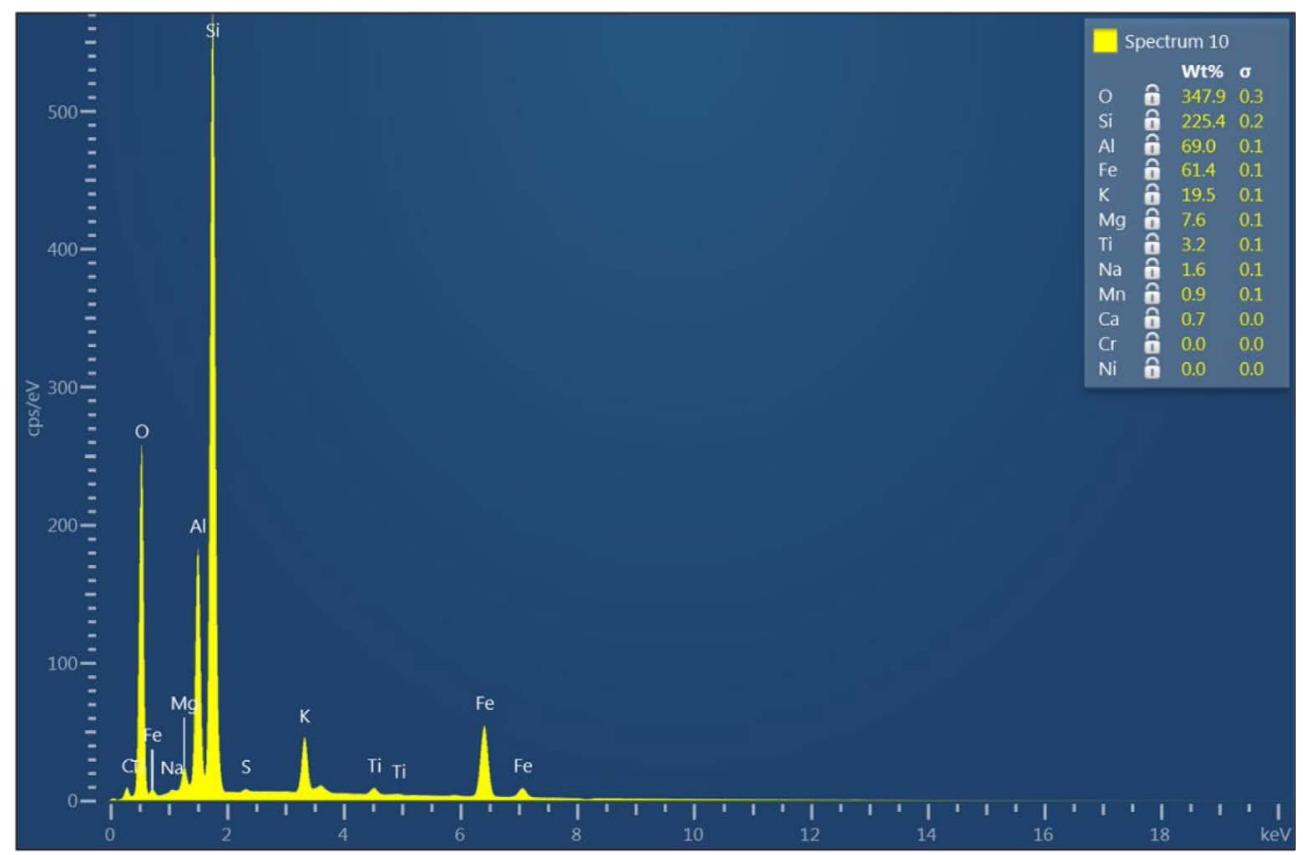

Fig. 6. Spectrum of the EDS map of the shale surface, showing element concentrations.

forces are measured from the locations on this grain. To show the importance of the localization of the analysis area, we measured adhesion forces not only on the quartz grain (spots 1, 2, 4, 5, 7, 8) but also at spots, which are at the slight offset from the right corner of the quartz inclusion (spots 3, $6,9)$. Spectra collected from the nine locations of the surface are presented in Table 1. Each location was measured three times.

One of the obtained cantilever deflection spectra, which was used to calculate the adhesion force between the surface and the cantilever, is presented in Figure 7. In this figure, dependance of the cantilever deflection on the distance from the tip to the surface ( $Z$ axis) can be observed. Here any adhesion force from $1.81 \mu \mathrm{m}$ to the sample surface could be registered. Whilst the figure shows $190 \mathrm{~nm}$ into the sample, the tip stays on the surface, where the only difference is in the increased applying force of the cantilever on the sample. The spectrum blue line represents the cantilever approaching the sample surface and the red line indicates retraction. During the retraction (right-to-left), the cantilever tip stays on the sample surface, until the gradually increasing force, applied by the motor becomes higher than the adhesion force, and the cantilever snaps back, which happened here at $55.5 \mathrm{nN}$. Here it can be seen in the constant decrease of the cantilever deflection from positive values (up to $51.3 \mathrm{nN}$, when cantilever was pushed down to the surface) to zero, and then to negative ones, which illustrate that the deflection is caused by the retention forces, holding the cantilever.

On average, registered adhesion force between the quartz grain on the shale surface and the cantilever tip at atmospheric conditions (spots $1,2,4,5,7,8$ ) was shown to be $56.5 \pm 5 \mathrm{nN}$. Meanwhile spots 3,6 and 9 in the offset, showed significantly lower values, varying from 30 to $41.7 \mathrm{nN}$, which demonstrates the importance of the preliminary topography
Table 1. Adhesion force values between the cantilever tip and shale surface, measured at nine locations (Fig. 3).

\begin{tabular}{lcccc}
\hline Spot & \multicolumn{3}{c}{ Measurement, nN } & Mineral \\
\hline 1 & 55.5 & 62.0 & 59 & Quartz \\
2 & 52.8 & 52.8 & 54.5 & Quartz \\
3 & 30.4 & 34.5 & 29.0 & Clay \\
4 & 63.7 & 63.7 & 58 & Quartz \\
5 & 47.8 & 54.6 & 55.2 & Quartz \\
6 & 42.2 & 39.2 & 43.7 & Clay \\
7 & 47.8 & 51.1 & 51.1 & Quartz \\
8 & 61.1 & 58.4 & 60.3 & Quartz \\
9 & 31.4 & 29.8 & 29.8 & Clay \\
\hline
\end{tabular}

imaging. Taking into account that approximate radius of the cantilever tip is $5 \mathrm{~nm}$, average adhesion between cantilever tip and quartz grain was calculated to be $0.36 \pm 0.03 \mathrm{nN} / \mathrm{nm}^{2}$.

According to Leite et al. [13], during retraction of the cantilever tip, the registered forces include: the adhesion between a sphere and a plane in vacuum, capillary forces of thin water layer between sample surface and the cantilever tip, as well as other forces that originate from specific binding. As can be seen from the blue line in Figure 7, there was no force registered while the tip approached the sample, which means that no electrostatic or van der Waals forces were observed. Moreover, as the tip is coated with $\mathrm{Au} / \mathrm{Ti}$, it is inert to the sample surface. Further, the experiment was not conducted in vacuum, and the only water present in the system was that which was adsorbed from the air onto the surfaces of the sample and the tip. Excluding the 


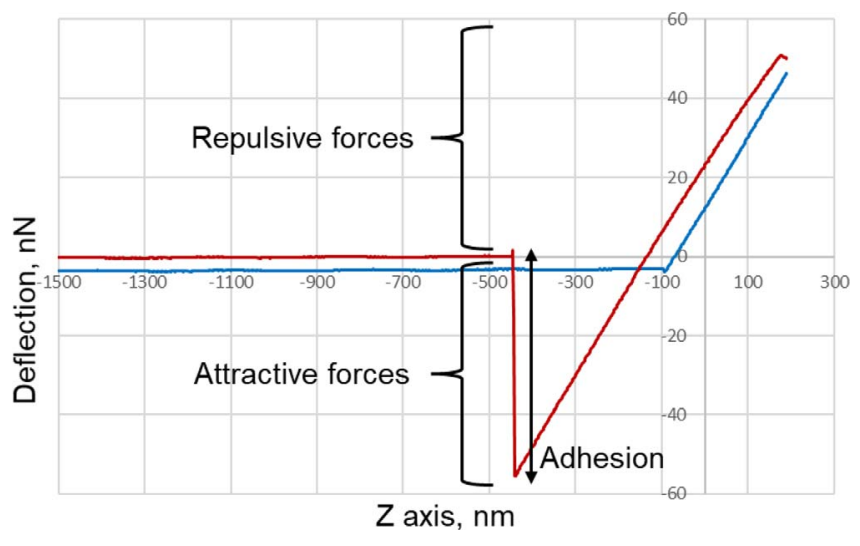

Fig. 7. An example of cantilever deflection spectrum. Blue line represents approaching the sample (left-to-right), while red lineretraction (right-to-left). Here, the maximum deflection of the cantilever before it was lifted from the sample surface was equal to the force of $55.2 \mathrm{nN}$.

mentioned forces, we state that the registered adhesion force in the experiment equals capillary force of the water meniscus, formed between sample surface and cantilever tip. During retraction, deflection of the cantilever was observed, which corresponds to an adhesion or capillary force of $55.2 \mathrm{nN}$.

In the literature the possibility of converting capillary force values to angle degrees (which are commonly used for wettability evaluation) was suggested, using the following formula [24]:

$$
F=4 \pi R \gamma \cos \theta
$$

$R$ - radius of tip curvature,

$\gamma$ - surface tension of water,

$\theta$ - angle between the sample surface and formed water meniscus.

However, here we observing much higher force values than expected from theory. This can be caused by two reasons. Firstly, this equation has only been experimentally confirmed for water meniscus radii larger than $5 \mathrm{~nm}$ [22, 24], while in our case the radius of the water meniscus is significantly smaller. This means that theoretical assumption of the size of formed water meniscus at this tip size is incorrect. Secondly, this theoretical equation does not take into account surface roughness of the sample, which affects surface of interaction, or relative humidity of the air.

\section{Conclusion}

In this study, the adhesion force values at the cantilever tip interaction with a quartz grain, inclined in the shale sample surface, have been measured using the AFM technique. Moreover, the importance of localizing the analysis area in highly heterogeneous samples, such as shales has been shown: without initial analysis of the surface topography, values can be obtained for different minerals even at micrometer scale. Meanwhile, SEM and EDS analyses were used to identify mineral composition of the shale sample surface and support the localization of the analysis area at nanoscale. The conclusions from this research study can presented as following:

- Conducting adhesion force measurement without preliminary characterization of the analyzed surface area by AFM topography and SEM imaging leads to inability to localize the analysis area. This is especially significant for heterogenous samples with small grain sizes, such as shale.

- For the first time, adhesion force between cantilever tip and quartz grain, located on the shale surface has been measured; average adhesion force was shown to be $56.6 \pm 5 \mathrm{nN}$, or $0.36 \pm 0.03 \mathrm{nN} / \mathrm{nm}^{2}$.

- We showed that the obtained values are higher than ones, expected from theory. This can be explained by the difference in the size of the formed water meniscus at this tip size $(5 \mathrm{~nm})$ with theoretical assumptions.

- Currently, the AFM technique is capable of providing information about the adhesion forces between cantilever and sample surface, and consequently wettability at nanoscale. Conventional wettability measurement methods such as Amott tests, USBM, NMR or even contact angle measurements cannot provide such resolution.

- At the same time, the AFM technique is still novel, and there are many gaps in the data and prospects for new discoveries are good. For instance, complications in the correct estimation of the interaction geometry, and thus formed liquid meniscus between the sample surface and cantilever prevents correlation between wetting properties of sample surface and measured adhesion force values.

Acknowledgments. Authors would like to acknowledge Nanosurf representative, Christian Loebbe, for consulting about AFM and Michael Stein for the assistance with language and style. Ultrahigh resolution SEM image of the cantilever tip was obtained in John de Laeter Centre, Curtin University. S. Shilobreeva participated according to Russian state assignment for Vernadsky Institute of Geochemistry and Analytical Chemistry, Russian Academy of Science. No funding from external organizations was received for this research.

\section{References}

1 Maugeri L. (2004) Oil: Never cry wolf. Why the petroleum age is far from over, Science 304, 1114-1115.

2 Morrow N.R. (1990) Wettability and its effect on oil recovery, J. Pet. Tech. 42, 1476-1484.

3 Ivanova A., Mitiurev N., Cheremisin A., Orekhov A., Vasiliev A., Hairullin M., Afanasiev I. (2018) Direct wettability characterization of the carbonate reservoirs using different microscopic techniques, in: 80th EAGE Conference and Exhibition, June 11-14, 2018, Copenhagen, Denmark, pp. 1-5.

4 Schmatz J., Urai J.L., Berg S., Ott H. (2015) Nanoscale imaging of pore-scale fluid-fluid-solid contacts in sandstone, Geophys. Res. Lett. 42, 2189-2195. 
5 Mahani H., Keya A.L., Berg S., Bartels W.-B., Nasralla R., Rossen W.R. (2015) Insights into the mechanism of wettability alteration by low-salinity flooding (LSF) in carbonates, Energy Fuels 29, 1352-1367.

6 Iglauer S. (2017) $\mathrm{CO}_{2}$-water-rock wettability: Variability, influencing factors, and implications for $\mathrm{CO} 2$ geostorage, Acc. Chem. Res. 50, 1134-1142.

7 Karymov M., Prochazka K., Mendenhall J., Martin T., Munk P., Webber S. (1996) Chemical attachment of polystyrole-block-poly(methacrylic acid) micelles on a silicon nitride surface, Langmuir 12, 20, 4748-4753.

8 Karymov M., Tomschik M., Leuba S., Caiafa P., Zlatanova J. (2001) DNA methylation-dependent chromatin fiber compaction in vivo and in vitro: requirement for linker histone, Fasseb J. 15, 14, 2631-2641.

9 Mori O., Imae T. (1997) AFM investigation of the adsorption process of bovine serum albumin on mica, Colloids Surf. B 9, 31-36.

10 Pericet-Camara R., Papastavrou G., Borkovec M. (2004) Atomic force microscopy study of the adsorption and electrostatic self-organization of poly(amidoamine) dendrimers in mica, Langmuir 20, 3264-3270.

11 Javadpour F., Farshi M.M., Amrein M. (2012) Atomic-force microscopy: A new tool for gas- shale characterization, J. Can. Pet. Technol. 51, 04, 236-243. SPE 161015.

12 Liu K., Ostadhassan M., Jabbari H., Bubach B. (2016) Potential application of atomic force microscopy in characterization of nano-pore structures of Bakken formation, in: Low Perm Symposium, 5-6 May 2016, Denver, Colorado, USA. SPE-180276- MS SPE.

13 Leite F.L., Bueno C.Cc, Da Roz A.L., Ziemath E.C., Oliveira O.N. (2012) Theoretical models for surface forces and adhesion and their measurement using atomic force microscopy, Int. J. Mol. Sci. 13, 12773-12856.

14 Yin X., Miller J.D. (2012) Wettability of kaolinite basal planes based on surface force measurements using atomic force microscopy, Miner 29, 1.
15 Seiedi O., Rahbar M., Nabipour M., Emadi M., Ghatee M., Ayatollahi S. (2011) Atomic force microscopy (AFM) investigation on the surfactant wettability alteration mechanism of aged mica mineral surfaces, Energy Fuels 25, $183-188$.

16 Hassenkam T., Skovbjerg L.L., Stipp S.L.S. (2009) Probing the intrinsically oil-wet surfaces of pores in North Sea chalk at subpore resolution, PNAS 106, 15, 6071-6076.

17 Deng Y., Xu L., Lu H., Wang H., Shi Y. (2018) Direct measurement of the contact angle of water droplet on quartz in a reservoir rock with atomic force microscopy, Chem. Eng. Sci. 177, 445-454.

18 Ivanova A., Mitiurev N., Cheremisin A., Orekhov A., Kamyshinsky R., Vasiliev A. (2019) Characterization of organic layer in oil carbonate reservoir rocks and its effect on microscale wetting properties, Sci. Rep. 9, 10667.

19 Yesufu-Rufai S., Marcelis F., Georgiadis A., Berg S., Rucker M., van Wunnik J., Luckham P. (2020) Atomic Force Microscopy (AFM) study of redox conditions in sandstones: Impact of wettability modification and mineral morphology, Colloids Surf. A 597, 124765.

20 Kumar K., Dao E., Mohanty K.K. (2005) AFM study of mineral wettability with reservoir oils, J Colloid Interface Sci 289, 206-217.

21 Sedin D., Rowlen K. (2000) Adhesion forces measured by atomic force microscopy in humid air, Anal. Chem. 72, 2183-2189.

22 Jones R., Pollock H., Cleaver J., Hodges C. (2002) Adhesion forces between glass and silicon surfaces in air studied by AFM: Effects of relative humidity, particle size, roughness and surface treatment, Langmuir 18, 8045-8055.

23 Mitiurev N., Verrall M., Ivanova A., Keshavarz A., Iglauer S. (2021) Sample preparation for rock wettability studies via atomic force microscopy, APPEA J. 61, 1, 216-223.

24 Fisher L., Israelachvili J. (1981) Experimental studies on the applicability of the Kelvin equation to highly curved concave menisci, J. Colloid Interface Sci. 80, 528-541. 